\title{
Tensor decomposition for bosonic and fermionic scattering amplitudes
}

\author{
Tiziano Peraro ${ }^{1, *}$ and Lorenzo Tancredi $\oplus^{2, \dagger}$ \\ ${ }^{1}$ Dipartimento di Fisica e Astronomia, Università di Bologna e INFN, \\ Sezione di Bologna, via Irnerio 46, I-40126 Bologna, Italy \\ ${ }^{2}$ Rudolf Peierls Centre for Theoretical Physics, University of Oxford, Clarendon Laboratory, \\ Parks Road, Oxford OXI 3PU, UK
}

(Received 16 December 2020; accepted 8 March 2021; published 29 March 2021)

\begin{abstract}
In this paper, we elaborate on a method to decompose multiloop multileg scattering amplitudes into Lorentz-invariant form factors, which exploits the simplifications that arise from considering fourdimensional external states. We propose a simple and general approach that applies to both fermionic and bosonic amplitudes and allows us to identify a minimal number of physically relevant form factors, which can be related one to one to the independent helicity amplitudes. We discuss explicitly its applicability to various four- and five-point scattering amplitudes relevant for LHC physics.
\end{abstract}

DOI: 10.1103/PhysRevD.103.054042

\section{INTRODUCTION}

In the computation of multiloop multileg scattering amplitudes in quantum field theory, one encounters at least two different types of problems, whose complexity increases quickly with the number of legs and the number of loops involved. First, one has to write down a loop integrand for the corresponding scattering process in a suitable form, and, second, one has to find a way to compute the relevant integrals efficiently, either analytically or numerically.

In this paper, we will focus on the first of the two problems. The standard approach starts from a representation for the scattering amplitude in terms of Feynman diagrams and requires one to perform a series of algebraic manipulations in order to separate the overall Lorentz structures (i.e., combinations of spinors, polarization vectors, etc.) from what are properly called scalar Feynman integrals. The Feynman integrals encompass the analytic dependence of the amplitude on the external kinematics, its branch cuts, and its divergences. While following this program might appear straightforward at first, it can become extremely cumbersome in practice for multiloop and multileg scattering amplitudes, where different sets of manipulations have to be performed on different diagrams in order to achieve the desired decomposition.

\footnotetext{
*tiziano.peraro@unibo.it

†lorenzo.tancredi@physics.ox.ac.uk
}

Published by the American Physical Society under the terms of the Creative Commons Attribution 4.0 International license. Further distribution of this work must maintain attribution to the author(s) and the published article's title, journal citation, and DOI. Funded by SCOAP ${ }^{3}$.
A common solution, which allows one to readily decompose any scattering amplitude in terms of scalar Feynman integrals, is the so-called projector method: Starting from the symmetries of the scattering amplitudes, in primis Poincaré and gauge invariance, one can write down a generic decomposition for any amplitude in terms of a basis of Lorentz tensors, multiplied by scalar form factors. The decomposition, being only based on symmetry considerations, is nonperturbative and holds at any number of loops. Starting from this, one can build projector operators which, once applied on the scattering amplitude, extract the corresponding form factors. Very importantly, since loop amplitudes are generically divergent in $d=4$ space-time dimensions, and dimensional regularization [1-3] is used throughout the computation of Feynman integrals, the aforementioned tensor and projector decomposition is usually performed in $d$ space-time dimensions. After the form factors have been computed, one can use them to compute the so-called helicity amplitudes for the process considered. In practice, one identifies the set of independent helicity amplitudes necessary to fully characterize the problem, and, starting from the generic $d$-dimensional tensor decomposition, one fixes the helicities of the external states, obtaining a representation for the helicity amplitudes in terms of linear combinations of the form factors. Clearly, in doing this, one has to switch from $d$ - to four-dimensional external states, moving from what is usually referred to as common dimensional regularization (CDR) to the so-called 't Hooft-Veltman scheme (tHV). ${ }^{1}$ In this step, one often realizes that only specific linear combinations of the original form factors appear in the

\footnotetext{
${ }^{1}$ See [4] and references therein for a discussion about different regularization schemes.
} 
physical helicity amplitudes, often leading to simplifications in the final result.

This method has been applied extremely successfully in the calculation of a countless scattering amplitudes for $2 \rightarrow 1$ and $2 \rightarrow 2$ processes; see, for example, [5-8]. Nevertheless, there are (at least) two main reasons why one might want to improve on this method. First of all, with the increase of the number of external legs, the number of $d$-dimensional tensors required is bound to increase very fast due to the combinatorics stemming from the large number of independent external momenta. Moreover, insisting on using $d$-dimensional external states, when dealing with external fermions, forces us to work with the $d$-dimensional algebra of gamma matrices which, differently from the four-dimensional one, is not closed. As we will see in an explicit example below, for some processes, this prevents us from even being able to provide a generic nonperturbative tensor decomposition valid at any number of loops. Finally, it seems reasonable to expect that if we were able to identify from the beginning those and only those (combinations of) form factors that contribute to the physical scattering amplitudes, their calculation could turn out to be simpler than that of the full, unphysical, $d$-dimensional ones.

This is not the first paper where this question has been addressed. In Ref. [9], a solution was proposed, which involves the computation of polarized scattering amplitudes in an hybrid dimensional regularization scheme. In the context of this method, a connection between helicity amplitudes and a four-dimensional treatment of tensors and projectors has also been highlighted in Ref. [10]. A different idea has been proposed in Ref. [11], where it has been shown that one can build projector operators that project directly onto the helicity amplitudes. The important finding of [11] was that, when applying this idea to the scattering of $n>4$ particles, the ensuing helicity projectors could be expressed as linear combinations of a physical subset of the standard $d$-dimensional tensors. Interestingly, the number of independent tensors would match the number of helicity amplitudes, pointing to the fact that the minimal complexity of the problem could be exposed. As a consequence, restricting to the subspace of physical four-dimensional tensors allows one to keep the complexity stemming from the large number of independent $d$-dimensional tensors under control, since their number is always bound by the number of helicity amplitudes in the problem.

Two important considerations were still missing in [11]: first, how to generalize those findings to (the, in principle, simpler case of) the scattering of $n \leq 4$ particles; and secondly, an efficient way to determine the basis of independent tensors in the fermionic case, which does not go through the enumeration of all $d$-dimensional tensor structures and the explicit construction of helicity projectors. In this paper, we provide a solution for these two remaining issues, describing a new generic approach to the decomposition of multiloop multileg Feynman amplitudes in the 't Hooft-Veltman scheme, which matches one to one the number of independent helicity amplitudes in the problem.

\section{THE GENERAL IDEA}

In order to describe the idea behind this paper, let us consider a generic process, where $n$ unspecified bosonic and/or fermionic particles collide. For definiteness, we could imagine to be working in QCD, but this is not necessary for the discussion that will follow. Using Lorentz invariance, gauge invariance, and any other symmetries of the problem at hand, we can parametrize the scattering amplitude for the process in terms of a basis of independent tensor structures $T_{i}$ as

$$
\mathcal{A}\left(p_{1}, \ldots, p_{n}\right)=\sum_{i=1}^{N} \mathcal{F}_{i}\left(p_{1}, \ldots, p_{n}\right) T_{i},
$$

where $\mathcal{F}_{i}$ are scalar form factors. The tensors $T_{i}$ are built using the polarization states of the external particles, respecting the Lorentz and gauge symmetries of the problem and assuming that external states are $d$-dimensional. The standard procedure would then consist in defining projector operators, decomposed in terms of the same basis tensors,

$$
\mathcal{P}_{j}=\sum_{i=1}^{N} c_{i}^{(j)}\left(d ; p_{1}, \ldots, p_{n}\right) T_{i}^{\dagger},
$$

such that, by applying them on the amplitude in Eq. (2.1) and summing over the polarizations of the external states, one finds

$$
\sum_{\text {pol }} \mathcal{P}_{j} \mathcal{A}\left(p_{1}, \ldots, p_{n}\right)=\mathcal{F}_{j}\left(p_{1}, \ldots, p_{n}\right) .
$$

The coefficients $c_{i}^{(j)}\left(d ; p_{1}, \ldots, p_{n}\right)$ in Eq. (2.2) can be computed by imposing that Eq. (2.3) is satisfied. This requires one, in general, to solve a system of $N$ equations in $N$ unknowns. Equivalently, it is convenient to define the matrix,

$$
M_{i j}=\sum_{\text {pol }} T_{i}^{\dagger} T_{j}
$$

such that the projectors can be obtained by computing its inverse,

$$
c_{i}^{(j)}\left(d ; p_{1}, \ldots, p_{n}\right)=\left(M^{-1}\right)_{i j} .
$$

We stress here that, in general, the solution for $c_{i}^{(j)}\left(d ; p_{1}, \ldots, p_{n}\right) \quad$ will depend on the space-time 
dimensional regulator $d$ and might, in particular, be singular as $d \rightarrow 4$.

Let us now go back to the amplitude in Eq. (2.1). In order to compute physical observables, we are ultimately interested in considering so-called helicity amplitudes, which are obtained by fixing the helicities of the external states in all possible ways. This can be done directly in Eq. (2.1), where the only dependence on the polarization of the external states resides in the tensors $T_{i}$. In particular, if the helicity of particle $i$ is $\lambda_{i}$, we can write

$\mathcal{A}^{\lambda_{1}, \ldots, \lambda_{n}}\left(p_{1}, \ldots, p_{n}\right)=\sum_{i=1}^{N} \mathcal{F}_{i}\left(p_{1}, \ldots, p_{n}\right) T_{i}^{\lambda_{1}, \ldots \lambda_{n}}$

Importantly, when we fix the helicities, we also specify the external states to be four-dimensional. This calculation scheme, where the form factors are computed in dimensional regularization but the external states are kept fourdimensional, corresponds to the so-called 't Hooft-Veltman scheme, where external momenta and polarizations are in four dimensions, while internal states and loop momenta are in $d$ dimensions. It is not difficult to imagine that when this is done, some of the tensors that used to be independent for generic $d$ can become linearly dependent of each other. It is easy to see that this should happen whenever the number of $d$-dimensional tensors $N$ is larger than the number of helicity amplitudes with four-dimensional external states. In this case, in fact, one could imagine promoting the linear combinations of tensors in Eq. (2.6) to new independent tensors, and one would expect that only those combinations should be enough to describe the helicity amplitudes. Here, it is important to stress that the counting of the independent helicity amplitudes slightly changes depending on the number of external legs in the process. Indeed, it is easy to convince oneself that, with $n \leq 4$ external particles in four dimensions, it is impossible to construct a parity-odd invariant free of spinor phases. This implies that the helicity amplitudes for such processes, up to an overall helicity-dependent spinor phase that does not depend on the loop order, have trivial behavior under parity transformation. This allows one to reduce the number of different helicity amplitudes by a factor of 2 . On the other hand, for $n \geq 5$ external particles, one can build parity-odd invariants, and the helicity amplitudes will depend in a nontrivial way on them. The typical example is the scattering of five massless partons in QCD where the amplitudes depend on the parity odd invariant tr $5=$ $\operatorname{tr}\left(\gamma_{5} \not \not_{1} \not \not_{2} \not \not_{3} \not \not_{4}\right)$. In this case, all helicities have to be treated as different.

We will see various examples of this in the following. For now, let us ignore the details and simply assume that, when four-dimensional external states are considered, only the first $Q \leq N$ tensors are independent, and they are sufficient to span the tensor space on which the scattering amplitude is defined. As we will see more explicitly later, one way the linear dependence manifests in our calculation is through the fact that the determinant of the matrix in Eq. (2.4) would go to zero as $d \rightarrow 4$. In order words, in $d=4$, the matrix $M$ would not be full rank; i.e., $\operatorname{rank}(M)=Q \leq N$. In this situation, we can always reorder the tensors such that the independent ones are the first $Q$,

$$
\bar{T}_{j}=T_{j}, \quad j=1, \ldots, Q .
$$

We also assume that the first $Q$ tensors are sufficient to describe the tensor space spanned by the external helicity states. It is then convenient to define $Q$ intermediate projector operators, given by

$$
\begin{aligned}
M_{i j}^{Q \times Q} & =\sum_{\text {pol }} \bar{T}_{i}^{\dagger} \bar{T}_{j}, \quad P_{i}^{Q \times Q}=\sum_{j=1}^{Q}\left(M^{Q \times Q}\right)_{i j}^{-1} \bar{T}_{j}^{\dagger}, \\
i, j & =1, \ldots, Q .
\end{aligned}
$$

The matrix $M_{i j}^{Q \times Q}$ is computed and inverted in $d$ dimensions, but, by construction, it has full rank even in the limit $d \rightarrow 4$.

At this point, we complete the basis of $d$-dimensional tensors by defining the remaining $N-Q$ tensors,

$$
\bar{T}_{i}=T_{i}-\sum_{j=1}^{Q}\left(P_{j}^{Q \times Q} T_{i}\right) \bar{T}_{j}, \quad i=Q+1, \ldots, N,
$$

where, once more, projections and sums over polarizations are performed in $d$ dimensions. The definition in Eq. (2.9) amounts to removing from the original $T_{i}$ their projection along the first $Q$ tensors. Of course, if we are only interested in the $d$-dimensional problem, it is always possible to perform such a redefinition of the basis of tensors, which will effectively block diagonalize the corresponding projectors. Indeed, we can now define

$\bar{M}_{i j}=\sum_{\mathrm{pol}} \bar{T}_{i}^{\dagger} \bar{T}_{j}, \quad \bar{P}_{i}=\sum_{j=1}^{N}(\bar{M})_{i j}^{-1} \bar{T}_{j}^{\dagger}, \quad i, j=1, \ldots, N$,

where the inverse of the matrix $\bar{M}$ will have, by construction, the block form,

$$
(\bar{M})_{i j}^{-1}=\left(\begin{array}{cc}
\left(M^{Q \times Q}\right)_{i j}^{-1} & 0 \\
0 & Y_{i j}
\end{array}\right) .
$$

Until now, we have performed all manipulations in $d$ dimensions and have not made explicit use of the fact that the first $Q$ tensors span the entire physical space required to compute the helicity amplitudes in four-dimensional external states. Let us go now back to Eq. (2.1). It is 
straightforward to reexpress it in terms of the new basis of tensors,

$$
\begin{aligned}
\mathcal{A}\left(p_{1}, \ldots, p_{n}\right)= & \sum_{i=1}^{Q} \mathcal{F}_{i}\left(p_{1}, \ldots, p_{n}\right) T_{i} \\
& +\sum_{i=Q+1}^{N} \mathcal{F}_{i}\left(p_{1}, \ldots, p_{n}\right) T_{i}, \\
= & \sum_{i=1}^{Q}\left[\mathcal{F}_{i}+\sum_{j=Q+1}^{N} \mathcal{F}_{j}\left(P_{i}^{Q \times Q} T_{j}\right)\right] \bar{T}_{i} \\
& +\sum_{j=Q+1}^{N} \mathcal{F}_{j} \bar{T}_{j}=\sum_{i=1}^{N} \overline{\mathcal{F}}_{i} \bar{T}_{i},
\end{aligned}
$$

where

$$
\overline{\mathcal{F}}_{i}= \begin{cases}\mathcal{F}_{i}+\sum_{j=Q+1}^{N} \mathcal{F}_{j}\left(P_{i}^{Q \times Q} T_{j}\right) & i=1, \ldots, Q \\ \mathcal{F}_{i} & i=Q+1, \ldots, N .\end{cases}
$$

From Eq. (2.13), we see that, as long as the matrix $M_{i j}^{Q \times Q}$ defined in Eq. (2.8) has full rank in $d=4$ (and, therefore, its inverse is finite in $d=4$ ), the new form factors $\overline{\mathcal{F}}_{i}$ are smooth linear combinations of the original form factors $\mathcal{F}_{i}$. We proceed now and fix the helicities on Eq. (2.12), finding

$\mathcal{A}^{\lambda_{1}, \ldots, \lambda_{n}}\left(p_{1}, \ldots, p_{n}\right)=\sum_{i=1}^{Q} \overline{\mathcal{F}}_{i}\left(p_{1}, \ldots, p_{n}\right) \bar{T}_{i}^{\lambda_{1}, \ldots \lambda_{n}}$,

where we stress that the sum runs only over the first $Q$ tensors. We stress that this is the crucial point; as long as the first $Q$ tensors span the full tensor space defined by the external states, the $N-Q$ remaining tensors defined in Eq. (2.9) are exactly zero for four-dimensional external states, and, in particular, they are zero when we fix the helicities of the external particles in all possible ways,

$$
\bar{T}_{i}^{\lambda_{1}, \ldots \lambda_{n}}=0, \quad i=Q+1, \ldots, N .
$$

From now on, we will refer to these tensors as irrelevant tensors and similarly to their projectors as irrelevant projectors, while the nonzero ones will be refereed to as relevant ones.

Let us pause here to stress what we have achieved: We have found a new decomposition of the amplitude, which allows us to extract the full information necessary for computing all physical helicity amplitudes from a minimal number of form factors, which multiply tensors that are independent in $d=4$ space-time dimensions. As we will see explicitly below, in our construction, it is crucial that the tensors that we pick span the full tensor space where the scattering amplitude is defined. Intuitively, we expect the dimension of this space to coincide with the number of different helicity amplitudes for the process considered, which implies that there is a one-to-one correspondence between the helicity amplitudes and our relevant tensor structures. Notice also that, since the matrix in Eq. (2.11) is in block form, the $Q$ physical form factors can be computed using projectors that are decomposed purely in terms of the $Q$ independent tensors. We never need to compute the contractions of the amplitude with the irrelevant tensors $\bar{T}_{i}$, $i=Q+1, \ldots, N$. While these properties are clearly appealing from an aesthetic point of view, depending on the complexity of the problem at hand, they might also constitute a source of substantial practical simplification.

In the next section, we will show how the formal construction works out in practice for different four- and five-point scattering processes, trying to elucidate all points discussed above.

\section{CASE OF STUDY: $g g \rightarrow g g$ SCATTERING}

We start by considering the scattering of four massless spin-1 bosons. For definiteness, we imagine dealing with four-gluon scattering, but this is not necessary for the arguments that follow. Let us consider the process,

$$
g\left(p_{1}\right)+g\left(p_{2}\right)+g\left(p_{3}\right)+g\left(p_{4}\right) \rightarrow 0,
$$

and call $\epsilon_{j}^{\mu}$ the polarization vector for to the gluon $j$. We define the usual Mandelstam invariants to be

$$
\begin{aligned}
& s=\left(p_{1}+p_{2}\right)^{2}, \quad t=\left(p_{1}+p_{3}\right)^{2}, \\
& u=\left(p_{2}+p_{3}\right)^{2}=-s-t .
\end{aligned}
$$

We indicate the scattering amplitude as $\mathcal{A}_{4 g}\left(p_{1}, p_{2}, p_{3}\right)$. Once stripped of the polarizations of the external gluons, this amplitude must be a rank-4 Lorentz tensor,

$\mathcal{A}_{4 g}\left(p_{1}, p_{2}, p_{3}\right)=\epsilon_{1, \mu} \epsilon_{2, \nu} \epsilon_{3, \rho} \epsilon_{4, \sigma} \mathcal{A}_{4 g}^{\mu \nu \rho \sigma}\left(p_{1}, p_{2}, p_{3}\right)$.

The most general Lorentz-covariant decomposition for a rank-4 tensor in $d$ space-time dimensions involves 138 tensor structures; see, for example, [12]. Imposing transversality for each external gluon $\epsilon_{i} \cdot p_{i}=0$, together with fixing the gauge, allows one to reduce these structures to 10 independent ones. In the case of gluon scattering, given the symmetry of the external states and the fact that color ordering can be used to isolate simpler primitive amplitudes, a convenient gauge choice is the cyclic one $\epsilon_{i} \cdot p_{i+1}=0$, where we identify $p_{5}=p_{1}$. With this choice, the 10 tensors read 


$$
\begin{aligned}
& T_{1}=\epsilon_{1} \cdot p_{3} \epsilon_{2} \cdot p_{1} \epsilon_{3} \cdot p_{1} \epsilon_{4} \cdot p_{2}, \\
& T_{2}=\epsilon_{1} \cdot p_{3} \epsilon_{2} \cdot p_{1} \epsilon_{3} \cdot \epsilon_{4}, \quad T_{3}=\epsilon_{1} \cdot p_{3} \epsilon_{3} \cdot p_{1} \epsilon_{2} \cdot \epsilon_{4}, \quad T_{4}=\epsilon_{1} \cdot p_{3} \epsilon_{4} \cdot p_{2} \epsilon_{2} \cdot \epsilon_{3}, \\
& T_{5}=\epsilon_{2} \cdot p_{1} \epsilon_{3} \cdot p_{1} \epsilon_{1} \cdot \epsilon_{4}, \quad T_{6}=\epsilon_{2} \cdot p_{1} \epsilon_{4} \cdot p_{2} \epsilon_{1} \cdot \epsilon_{3}, \quad T_{7}=\epsilon_{3} \cdot p_{1} \epsilon_{4} \cdot p_{2} \epsilon_{1} \cdot \epsilon_{2}, \\
& T_{8}=\epsilon_{1} \cdot \epsilon_{2} \epsilon_{3} \cdot \epsilon_{4}, \quad T_{9}=\epsilon_{1} \cdot \epsilon_{4} \epsilon_{2} \cdot \epsilon_{3}, \quad T_{10}=\epsilon_{1} \cdot \epsilon_{3} \epsilon_{2} \cdot \epsilon_{4} .
\end{aligned}
$$

Following the notation introduced in Sec. II, we then write the amplitude for the scattering of four gluons as

$$
\mathcal{A}_{4 g}\left(p_{1}, p_{2}, p_{3}\right)=\sum_{j} \mathcal{F}_{j}\left(p_{1}, p_{2}, p_{3}\right) T_{j}
$$

where $F_{j}$ are the 10 scalar form factors. In CDR (i.e., keeping the external polarizations in $d$ dimensions), these 10 tensors are independent, and they must be kept as they are. As described in the previous section, the standard approach would then be to derive 10 projectors that single out each of the form factors.

Nevertheless, we have seen that as long as not all tensors are independent for four-dimensional external states, this procedure can be simplified. A first counting of the number of helicities involved shows immediately that we expect $2^{4}=16$ helicities, which are reduced to eight by parity (we recall, from the previous section, that this reduction by parity holds up to $n=4$ external legs). We expect, therefore, to be able to describe the physical process in terms of eight independent tensor structures. Indeed, two of the tensors in Eq. (3.4) do become linear dependent when the external states are specialized in four dimensions. This can be demonstrated in several ways. One possibility is to explicitly project the 10 tensors in Eq. (3.4) onto a complete set of independent external four-dimensional helicity states (for example, using the spinor helicity formalism).

As hinted to in Sec. II, an alternative way to see this is to study the rank of the matrix,

$$
M_{i j}=\sum_{\mathrm{pol}} T_{i}^{\dagger} T_{j}
$$

where, in the case under study, the sum over polarizations has to be performed respecting

$$
\sum_{\mathrm{pol}} \epsilon_{i}^{\mu} \epsilon_{i}^{\nu}=-g^{\mu \nu}+\frac{p_{i}^{\mu} p_{i+1}^{\nu}+p_{i}^{\nu} p_{i+1}^{\mu}}{p_{i} \cdot p_{i+1}}
$$

In the standard approach, the 10 projectors that single out the 10 form factors $F_{j}$ would then be obtained by inverting the matrix $M_{i j}$. If one attempts to do this in $d=4$ spacetime dimensions, one finds that $M_{i j}$ is not full rank and, in particular, $\operatorname{rank}\left(M_{i j}\right)=8$. This implies, as expected, that only eight tensors are independent when external states are taken in four dimensions. In particular, in order to cover the physical space defined by the external polarizations, we consider the first seven tensors $T_{1}, \ldots, T_{7}$ and the symmetric combination $T_{8}+T_{9}+T_{10}$. While one can intuitively understand this to be a good choice due to symmetry reasons, we also show how construct the basis in a more systematic way in the Appendix A.

Let us then follow Sec. II and define

$$
\begin{aligned}
\bar{T}_{i} & =T_{i}, \quad i=1, \ldots, 7 \\
\bar{T}_{8} & =T_{8}+T_{9}+T_{10} \\
M_{i j}^{(8 \times 8)} & =\sum_{\text {pol }} \bar{T}_{i}^{\dagger} \bar{T}_{j},
\end{aligned}
$$

where we still perform all polarization sums in $d$ dimensions to compute $M_{i j}^{(8 \times 8)}$. The matrix $M_{i j}^{(8 \times 8)}$ is now invertible in $d=4$ dimensions. Keeping still generic dependence on $d$, its inverse can be easily computed to be

$$
\left(M^{(8 \times 8)}\right)_{i j}^{-1}=\frac{1}{3(d-1)(d-3) t^{2}}\left(X_{i j}^{(0)}+d X_{i j}^{(1)}\right),
$$

with

$$
X_{i j}^{(0)}=\left(\begin{array}{ccc}
-\frac{8\left(s^{2}-4 s u+u^{2}\right)}{s^{2} u^{2}} & \frac{2 t}{s u} & \frac{4\left(t^{2}+5 t u+3 u^{2}\right)}{s u^{2}} \\
\frac{2 t}{s u} & -2 & \frac{s+2 u}{u} \\
\frac{4\left(t^{2}+5 t u+3 u^{2}\right)}{s u^{2}} & \frac{s+2 u}{u} & -\frac{2\left(t^{2}+4 t u+u^{2}\right)}{u^{2}} \\
-\frac{2 t}{s u} & -1 & \frac{t-u}{u} \\
\frac{2 t}{s u} & 1 & \frac{s+2 u}{u} \\
\frac{4\left(s^{2}+3 s u-u^{2}\right)}{s^{2} u} & \frac{2 t+u}{s} & -\frac{(2 s+u)(s+2 u)}{s u} \\
-\frac{2 t}{s u} & -1 & \frac{t-u}{u} \\
\frac{-2 t^{2}+3 t u+3 u^{2}}{s u} & s+u & \frac{t(t-u)}{u}
\end{array}\right.
$$$$
\left.\begin{array}{ccccc}
-\frac{2 t}{s u} & \frac{2 t}{s u} & \frac{4\left(s^{2}+3 s u-u^{2}\right)}{s^{2} u} & -\frac{2 t}{s u} & \frac{-2 t^{2}+3 t u+3 u^{2}}{s u} \\
-1 & 1 & \frac{2 t+u}{s} & -1 & s+u \\
\frac{t-u}{u} & \frac{s+2 u}{u} & -\frac{(2 s+u)(s+2 u)}{s u} & \frac{t-u}{u} & \frac{t(t-u)}{u} \\
-2 & -1 & \frac{2 s+u}{s} & 1 & t \\
-1 & -2 & \frac{2 t+u}{s} & -1 & s+u \\
\frac{2 s+u}{s} & \frac{2 t+u}{s} & \frac{2\left(2 s^{2}-2 t u-3 u^{2}\right)}{s^{2}} & \frac{2 s+u}{s} & -\frac{t(2 t+u)}{s} \\
1 & -1 & \frac{2 s+u}{s} & -2 & t \\
t & s+u & -\frac{t(2 t+u)}{s} & t & t^{2}
\end{array}\right)
$$ 


$$
X_{i j}^{(1)}=\left(\begin{array}{cccccccc}
\frac{3(d+2)}{t^{2}}+\frac{12}{s^{2}}-\frac{12}{s u}+\frac{12}{u^{2}} & -\frac{3}{s+u} & \frac{3 s(2 s+u)}{t u^{2}} & \frac{3}{s+u} & -\frac{3}{s+u} & \frac{3 u(s+2 u)}{s^{2}(s+u)} & \frac{3}{s+u} & 0 \\
-\frac{3}{s+u} & 3 & 0 & 0 & 0 & 0 & 0 & 0 \\
\frac{3 s(2 s+u)}{t u^{2}} & 0 & \frac{3 s^{2}}{u^{2}} & 0 & 0 & 0 & 0 & 0 \\
\frac{3}{s+u} & 0 & 0 & 3 & 0 & 0 & 0 & 0 \\
-\frac{3}{s+u} & 0 & 0 & 0 & 3 & 0 & 0 & 0 \\
\frac{3 u(s+2 u)}{s^{2}(s+u)} & 0 & 0 & 0 & 0 & \frac{3 u^{2}}{s^{2}} & 0 & 0 \\
\frac{3}{s+u} & 0 & 0 & 0 & 0 & 0 & 3 & 0 \\
0 & 0 & 0 & 0 & 0 & 0 & 0 & 0
\end{array}\right) .
$$

This matrix allows us to define eight intermediate projectors,

$$
P_{i}^{(8 \times 8)}=\sum_{j=1}^{8}\left(M_{i j}^{(8 \times 8)}\right)^{-1} \bar{T}_{j}^{\dagger},
$$

which can now by used to find the last two orthogonal tensors $\bar{T}_{9}$ and $\bar{T}_{10}$, following the prescription given in Eq. (2.9). This just amounts to removing their projection along the independent tensors as follows:

$$
\begin{gathered}
\bar{T}_{9}=T_{9}-\sum_{i=1}^{8}\left(P_{i}^{(8 \times 8)} T_{9}\right) \bar{T}_{i}, \\
\bar{T}_{10}=T_{10}-\sum_{i=1}^{8}\left(P_{i}^{(8 \times 8)} T_{10}\right) \bar{T}_{i} .
\end{gathered}
$$

As long as the first eight tensors $\bar{T}_{1}, \ldots, \bar{T}_{8}$ cover the full space spanned by the external polarizations, we can forget about these last two tensors $\bar{T}_{9}$ and $\bar{T}_{10}$ for any physical calculation with four-dimensional external helicity states. We can easily prove this by showing that $\bar{T}_{9}$ and $\bar{T}_{10}$ vanish when contracted with external helicity states, combined with the fact that - by construction-the last two tensors are completely orthogonal to the first eight. Indeed, if we insist in considering all 10 tensors $\bar{T}_{i}$ and define the matrix,

$$
\bar{M}_{i j}=\sum_{\mathrm{pol}} \bar{T}_{i}^{\dagger} \bar{T}_{j}, \quad \text { for } i, j=1, \ldots, 10,
$$

its inverse can be easily computed to be

$$
(\bar{M})_{i j}^{-1}=\left(\begin{array}{ccc}
\left(M^{(8 \times 8)}\right)_{i j}^{-1} & 0 & 0 \\
0 & \frac{2}{(d-3)(d-4)} & \frac{1}{(d-3)(d-4)} \\
0 & \frac{1}{(d-3)(d-4)} & \frac{2}{(d-3)(d-4)}
\end{array}\right) .
$$

As expected, the matrix is in block-diagonal form, such that the two dependent projectors (and tensors) completely decouple from the relevant ones.

It is thus straightforward to check that the tensors $\bar{T}_{1}, \ldots, \bar{T}_{8}$ form a complete physical basis by fixing the helicities of the gluons in all possible ways and showing that the two additional tensors always evaluate to zero. In other words, we have

$$
\bar{T}_{9}^{\lambda_{1}, \lambda_{2}, \lambda_{3}, \lambda_{4}}=\bar{T}_{10}^{\lambda_{1}, \lambda_{2}, \lambda_{3}, \lambda_{4}}=0
$$

for any choice of the external helicities. As before, we indicate with $\lambda_{i}= \pm$ the helicity of the $i$ th gluon. In particular, we can use the spinor-helicity formalism and parametrize the polarization vector of gluon $i$ as follows:

$$
\epsilon_{i,+}^{\mu}=\frac{\left\langle q_{i}\left|\gamma^{\mu}\right| i\right]}{\sqrt{2}\left\langle q_{i} i\right\rangle}, \quad \epsilon_{i,-}^{\mu}=\frac{\left[q_{i}\left|\gamma^{\mu}\right| i\right\rangle}{\sqrt{2}\left\langle i q_{i}\right\rangle},
$$

where $q_{i}$ is the gauge fixing four-momentum, which is chosen respecting Eq. (3.6). Equations (3.13) can thus be shown to be correct for any helicity configuration. A simple way to do this is by means of the momentum twistor parametrization for the spinor products $[13,14]$. This proves that the tensors $\bar{T}_{9}$ and $\bar{T}_{10}$ are irrelevant, and thus, $\bar{T}_{1}, \ldots, \bar{T}_{8}$ form a complete basis of physical tensors for all helicity amplitudes with four-dimensional external states.

Before moving to the next example, let us see how we expect the construction to generalize if we consider massive external bosons instead of massless ones, i.e., for example for the production of two $Z / W$ bosons in gluon fusion $g g \rightarrow Z Z$ or $g g \rightarrow W^{+} W^{-}$. If we limit ourselves to vectorlike coupling (which is enough if we are only interested in massless quark loops up to two loops; see, for example, the discussion in $[15,16])$, there are 20 independent tensor structures in $d$ dimensions. On the other hand, having two gluons and two massive vector bosons and taking parity invariance under account tells us that there should be $\left(2^{2} \times 3^{2}\right) / 2=18$ independent tensor structures when only four-dimensional external states are considered. This 
matches indeed the 18 independent combinations of form factors, which were identified a posteriori in $[15,16]$. We do not construct the tensors explicitly here, but we want to stress that, more recently, the same number of independent structures has been obtained with a different approach in [17]. There, the 20 independent tensors have been orthonormalized in $d=4$ using the well-known GramSchmidt procedure, which has allowed the authors to construct 18 independent tensors in the limit $d \rightarrow 4$.

\section{EXAMPLE 2: $q \bar{q} \rightarrow g g$ SCATTERING}

Let us repeat the same exercise for the production of two gluons in $q \bar{q}$ annihilation,

$$
q\left(p_{1}\right)+\bar{q}\left(p_{2}\right)+g\left(p_{3}\right)+g\left(p_{4}\right) \rightarrow 0 .
$$

Since all external states are massless, we use the same Mandelstam invariants defined in Eq. (3.2). We start again from the generic $d$-dimensional decomposition for the amplitude and impose transversality for the external gluons $\epsilon_{i} \cdot p_{i}=0$, with $i=3,4$. In order to further simplify the problem, we fix the gauge for the external gluons, such that $\epsilon_{3} \cdot p_{2}=\epsilon_{4} \cdot p_{1}=0$, which implies for the gluons the polarization sums,

$$
\begin{aligned}
& \sum_{\mathrm{pol}} \epsilon_{3}^{\mu} \epsilon_{3}^{\nu}=-g^{\mu \nu}+\frac{p_{3}^{\mu} p_{2}^{\nu}+p_{3}^{\nu} p_{2}^{\mu}}{p_{2} \cdot p_{3}}, \\
& \sum_{\mathrm{pol}} \epsilon_{4}^{\mu} \epsilon_{4}^{\nu}=-g^{\mu \nu}+\frac{p_{4}^{\mu} p_{1}^{\nu}+p_{4}^{\nu} p_{1}^{\mu}}{p_{2} \cdot p_{3}} .
\end{aligned}
$$

With this choice, we are left with five independent tensors structures in $d$ dimensions. A common choice is [6]

$$
\begin{aligned}
T_{1} & =\bar{u}\left(p_{2}\right) \phi_{3} u\left(p_{1}\right) \epsilon_{4} \cdot p_{2}, \\
T_{2} & =\bar{u}\left(p_{2}\right) \phi_{4} u\left(p_{1}\right) \epsilon_{3} \cdot p_{1}, \\
T_{3} & =\bar{u}\left(p_{2}\right) \not p_{3} u\left(p_{1}\right) \epsilon_{3} \cdot p_{1} \epsilon_{4} \cdot p_{2}, \\
T_{4} & =\bar{u}\left(p_{2}\right) \phi_{4} \not \not_{3} \phi_{3} u\left(p_{1}\right), \\
T_{5} & =\bar{u}\left(p_{2}\right) \phi_{3} \not \not_{3} \phi_{4} u\left(p_{1}\right) .
\end{aligned}
$$

Now, if we count the different helicities for the process, we get $2^{3}=8$, which should be divided by 2 due to parity invariance, such that we expect to have four helicities and four different tensors to describe them. We then expect that one of the tensors above should be linearly dependent when four-dimensional external states are considered. In order to verify that this is the case, we define the matrix,

$$
M_{i j}=\sum_{\mathrm{pol}} T_{i}^{\dagger} T_{j}
$$

where all polarization sums are done in $d$ dimensions as in Eq. (4.1) and verify easily that it is indeed not full rank in $d=4$, where $\operatorname{rank}\left(M_{i j}\right)=4$ instead. In order to find a subset of tensors that spans the full physical space, it is convenient to trade $T_{4}$ and $T_{5}$ for their symmetric combination and take as four independent tensors,

$\bar{T}_{i}=T_{i}, \quad i=1, \ldots, 3, \quad \bar{T}_{4}=\bar{u}\left(p_{2}\right) \not p_{3} u\left(p_{1}\right) \epsilon_{3} \cdot \epsilon_{4}$.

The fact that this is indeed the right combination can be justified by a similar argument as the one used to pick the eighth tensor in Eq. (3.7) (see also the discussion in the appendices).

As before, in order to define the fifth tensor $\bar{T}_{5}$, we start from the matrix,

$$
M_{i j}^{4 \times 4}=\sum_{\mathrm{pol}} \bar{T}_{i}^{\dagger} \bar{T}_{j}, \quad i, j=1, \ldots, 4,
$$

whose inverse reads

$$
\begin{aligned}
\left(M^{4 \times 4}\right)_{i j}^{-1}= & \frac{X_{i j}}{(d-3)(s+u)}, \\
X_{i j} & =\left(\begin{array}{cccc}
-\frac{u}{2 s^{2}} & 0 & -\frac{u}{2 s^{2}(s+u)} & 0 \\
0 & -\frac{u}{2 s^{2}} & \frac{u}{2 s^{2}(s+u)} & 0 \\
-\frac{u}{2 s^{2}(s+u)} & \frac{u}{2 s^{2}(s+u)} & -\frac{d u^{2}+4 s^{2}+4 s u}{2 s^{2} u(s+u)^{2}} & \frac{2 s+u}{2 s u(s+u)} \\
0 & 0 & \frac{2 s+u}{2 s u(s+u)} & -\frac{1}{2 u}
\end{array}\right) .
\end{aligned}
$$

We then use this matrix to define the four intermediate projectors,

$$
P_{i}^{4 \times 4}=\sum_{j=1}^{4}\left(M_{i j}^{(4 \times 4)}\right)^{-1} \bar{T}_{j}^{\dagger},
$$

such that the fifth orthogonal (irrelevant) tensor can be chosen as

$$
\bar{T}_{5}=T_{5}-\sum_{i=1}^{4}\left(P_{i}^{4 \times 4} T_{5}\right) \bar{T}_{i} .
$$

Once more, this achieves effectively a block decomposition of the projector matrix, which now reads

$$
\begin{array}{r}
\bar{M}_{i j}=\sum_{\mathrm{pol}} \bar{T}_{i}^{\dagger} \bar{T}_{j}, \quad \text { for } i, j=1, \ldots, 5, \\
(\bar{M})_{i j}^{-1}=\frac{1}{(d-3)(s+u)}\left(\begin{array}{cc}
X_{i j} & 0 \\
0 & -\frac{1}{2 u(d-4)}
\end{array}\right) .
\end{array}
$$

Note that this ensures that we only need to perform four contractions and not five in order to obtain the full information required to reconstruct the helicity amplitudes. For completeness, we write down the explicit expression for the fifth tensor in terms of the original ones, 


$$
\bar{T}_{5}=T_{5}-\frac{u}{s} T_{1}+\frac{u}{s} T_{2}-\frac{2}{s} T_{3}+T_{4},
$$

which is identically zero when evaluated for fourdimensional external states for any combination of helicities. Recently, these projector operators have been successfully applied for the calculation of the three-loop QCD corrections to the production of two photons in quark-antiquark annihilation [18].

\section{EXAMPLE 3: $q \bar{q} \rightarrow Q \bar{Q}$ SCATTERING}

The attentive reader might be rather disappointed at this point. In the previous two examples, we have indeed achieved a simplification, which, nevertheless, appears to be mainly of aesthetic nature; going from 10 to eight tensors for four-gluon scattering or from five to four for $q \bar{q} \rightarrow g g$ does not seem to be impressive. As we will see in the next sections, more impressive simplifications happen when considering the scattering of $n \geq 5$ particles. Moreover, for what concerns four-particle scattering, the fact that the new projectors are smooth as $d \rightarrow 4$ and their number matches one to one the number of independent helicity amplitudes could be already a reason for satisfaction. Nevertheless, in order to convince also the most demanding reader that this approach is worth pursuing and conceptually more appropriate also when dealing with fourparticle scattering, in this section, we discuss a four-point scattering amplitude where substantial simplifications can be achieved.

The prototypical example of what we want to show is the production of a pair of quarks in $q \bar{q}$ scattering,

$$
q\left(p_{1}\right)+\bar{q}\left(p_{2}\right)+Q\left(p_{3}\right)+\bar{Q}\left(p_{4}\right) \rightarrow 0 .
$$

This example is particularly interesting because, if one insists in working in CDR, it is not possible to find a finite number of tensor structures that span the whole space at every number of loops. The reason is simply that the algebra of the $\gamma$ matrices in $d$ dimensions is not closed. Indeed, following the standard approach to compute $q \bar{q} Q \bar{Q}$ scattering up to two loops, the following six tensor structures would be needed $[7,19]$ :

$T_{1}=\bar{u}\left(p_{2}\right) \gamma_{\mu_{1}} u\left(p_{1}\right) \bar{u}\left(p_{4}\right) \gamma^{\mu_{1}} u\left(p_{3}\right)$,

$T_{2}=\bar{u}\left(p_{2}\right) \not p_{3} u\left(p_{1}\right) \bar{u}\left(p_{4}\right) \not p_{1} u\left(p_{3}\right)$,

$T_{3}=\bar{u}\left(p_{2}\right) \gamma_{\mu_{1}} \gamma_{\mu_{2}} \gamma_{\mu_{3}} u\left(p_{1}\right) \bar{u}\left(p_{4}\right) \gamma^{\mu_{1}} \gamma^{\mu_{2}} \gamma^{\mu_{3}} u\left(p_{3}\right)$,

$T_{4}=\bar{u}\left(p_{2}\right) \gamma_{\mu_{1}} \not p_{3} \gamma_{\mu_{3}} u\left(p_{1}\right) \bar{u}\left(p_{4}\right) \gamma^{\mu_{1}} \not p_{1} \gamma^{\mu_{3}} u\left(p_{3}\right)$,

$T_{5}=\bar{u}\left(p_{2}\right) \gamma_{\mu_{1}} \gamma_{\mu_{2}} \gamma_{\mu_{3}} \gamma_{\mu_{4}} \gamma_{\mu_{5}} u\left(p_{1}\right) \bar{u}\left(p_{4}\right) \gamma^{\mu_{1}} \gamma^{\mu_{2}} \gamma^{\mu_{3}} \gamma^{\mu_{4}} \gamma^{\mu_{5}} u\left(p_{3}\right)$,

$T_{6}=\bar{u}\left(p_{2}\right) \gamma_{\mu_{1}} \gamma_{\mu_{2}} \not \gamma_{3} \gamma_{\mu_{4}} \gamma_{\mu_{5}} u\left(p_{1}\right) \bar{u}\left(p_{4}\right) \gamma^{\mu_{1}} \gamma^{\mu_{2}} \not p_{1} \gamma^{\mu_{4}} \gamma^{\mu_{5}} u\left(p_{3}\right)$,

but more would be needed at a higher number of loops.
It should be clear that all of these structures cannot be independent when the external states are four-dimensional. Indeed, as argued more extensively in Appendix B, it turns out that one only needs to consider tensors involving spinor chains, which are independent in four dimensions. In particular, the six structures presented above can be all related to the first two tensors $T_{1}$ and $T_{2}$ by use of fourdimensional Fiertz identities; hence, we only need to consider $T_{1}$ and $T_{2}$. Alternatively, we can also start with the full set of tensors and, using the same procedure illustrated in the previous sections, show that they can be replaced with only two physical tensors when external four-dimensional states are specified. Indeed, let us define once more the matrix,

$$
M_{i j}=\sum_{\text {pol }} T_{i}^{\dagger} T_{j}
$$

It is easy to verify that in $d=4$, the $\operatorname{rank}\left(M_{i j}\right)=2$. We define, therefore, the two independent tensors,

$$
\bar{T}_{i}=T_{i}, \quad i=1,2,
$$

and the $2 \times 2$ matrix,

$$
M_{i j}^{2 \times 2}=T_{i}^{\dagger} T_{j}, \quad i, j=1,2,
$$

which now has a smooth inverse in $d=4$,

$$
\begin{aligned}
\left(M^{2 \times 2}\right)_{i j}^{-1} & =\frac{1}{d-3} X_{i j}, \\
X_{i j} & =\left(\begin{array}{cc}
\frac{1}{4 s^{2}} & \frac{s+2 u}{4 s^{2} u(s+u)} \\
\frac{s+2 u}{4 s^{2} u(s+u)} & \frac{d s^{2}-2 s^{2}+4 s u+4 u^{2}}{4 s^{2} u^{2}(s+u)^{2}}
\end{array}\right) .
\end{aligned}
$$

It is worth comparing these expressions with the standard $d$-dimensional projectors derived in Eq. (2.15) of [7] to appreciate the gain in simplicity.

As for the previous examples, we use this matrix to define the intermediate projectors,

$$
P_{i}^{2 \times 2}=\sum_{j=1}^{2}\left(M_{i j}^{(2 \times 2)}\right)^{-1} \bar{T}_{j}^{\dagger},
$$

which allows us to decouple the irrelevant tensors by the projection,

$$
\bar{T}_{i}=T_{i}-\sum_{j=1}^{2}\left(P_{j}^{2 \times 2} T_{i}\right) \bar{T}_{j}, \quad \text { for } i \geq 3 .
$$

For the computation of the helicity amplitudes, all these extra tensors can be neglected. We stress once more that, if we insisted in using the $d$-dimensional tensors, their number would increase with the number of loops, while 
with our approach two tensors and two form factors is sufficient to obtain the helicity amplitudes at any number of loops. Finally, we can verify that the irrelevant tensors are all zero when we fix the polarizations of the external quarks in all possible ways, as expected.

\section{TENSOR DECOMPOSITION FOR $\boldsymbol{n}$-POINT AMPLITUDES}

The upshot of the previous sections can be summarized as follows: In order to exploit the simplifications that arise from treating external states in four space-time dimensions in the 't Hooft-Veltman scheme, one only needs identify how many tensor structures remain independent when fourdimensional external states are considered. We have seen that this number corresponds to the number of independent helicity amplitudes for the process studied. Then, for all practical purposes, one can simply throw away all the linearly dependent ones, which we referred to as irrelevant tensor structures. We have, in fact, shown, both in complete generality and with many explicit examples, that these irrelevant tensors can be decoupled from the relevant ones through a redefinition of the tensor basis, which amounts to choosing irrelevant tensors that are zero when evaluated for any combination of helicity for the external states. Therefore, the only nontrivial step in extending this procedure to the scattering of $n \geq 5$ particles consists in determining a priori which tensors are independent in four dimensions. In principle, one could, of course, start with the full set of $d$-dimensional tensors $T_{j}$ and study the rank of the corresponding projector matrix $M_{i j}$ in Eq. (2.4). While this is doable, it can become very soon impractical due to obvious combinatorics arguments.

Fortunately, it turns out that we do not need to go through the enumeration of the $d$-dimensional tensor structures at all for $n \geq 5$ scattering. The crucial point is that, starting at five points, the external momenta naturally provide us with four independent vectors to parametrize the helicity amplitudes. This, combined with the fact that only spinor chains that are independent in $d=4$ are needed (see Appendix B), allows us to decompose every Lorentz covariant object that appears in the calculation in terms of the of four independent four-dimensional external momenta and determine the independent tensors using four-dimensional algebra only. This is not true for $n \leq 4$, where we only have three or fewer independent momenta, and we need to introduce extra tensorial structures (as $\gamma^{\mu}, g_{\mu \nu}$ etc.) in order to be able to span the full fourdimensional space.

The simplest case is the scattering of five or more spin-1 massless particles and was treated in detail in [11]. In that case, it is immediate to see that the amplitude must be a rank-5 (or higher) tensor, made of the four independent momenta $p_{1}^{\mu}, \ldots, p_{4}^{\mu}$, which implies the existence of $4^{5}$ different tensor structures. Applying transversality plus a gauge choice for each external gluon, allows us to go down to $2^{5}=32$ tensor structures, which equals the number of independent helicities. In [11], it was shown explicitly that, by projecting on the physical helicity amplitudes, all extra $d$-dimensional tensor structures would not contribute and could be neglected. Note that here, unlike the four-point case, we cannot use parity to reduce the number of independent helicity amplitudes by a factor of 2 . The reason is that, starting at five points, the helicity amplitudes depend on the parity odd invariant $\operatorname{tr} 5=\operatorname{tr}\left(\gamma_{5} \not \not{ }_{1} \not \not_{2} \not \not_{3} \not \not_{4}\right)$. More explicitly, focusing on five-gluon scattering for definiteness, each helicity amplitude can be separated into a parity-even and a parity-odd part,

$$
\begin{aligned}
\mathcal{A}_{5 g}^{\lambda_{1}, \ldots, \lambda_{5}}\left(p_{1}, \ldots, p_{4}\right)= & \mathcal{A}_{+}^{\lambda_{1}, \ldots, \lambda_{5}}\left(p_{1}, \ldots, p_{4}\right) \\
& +\operatorname{tr} 5 \mathcal{A}_{-}^{\lambda_{1}, \ldots, \lambda_{5}}\left(p_{1}, \ldots, p_{4}\right),
\end{aligned}
$$

where we indicated with $\mathcal{A}_{5 g}^{\lambda_{1}, \ldots, \lambda_{5}}\left(p_{1}, \ldots, p_{4}\right)$ the amplitude for the scattering of five gluons of momenta $p_{j}$ and helicities $\lambda_{j}$. The fact that these amplitudes transform nontrivially under parity, even after dividing them by an overall spinor phase, does not allow us to restrict our tensor basis to 16 tensors only. Clearly, the same type of argument can be generalized for the scattering of five or more particles of any type. In what follows, we will show how to obtain a minimal number of four-dimensional tensor structures for different types of massless and massive five-point scattering amplitudes, which match the number of corresponding helicity amplitudes.

\section{A. Example 1: $q \bar{q} \rightarrow g g g$ scattering}

We start with the production of three spin-1 massless bosons from a spinor pair. For definiteness, we identify them with gluons, but, clearly, the same considerations would apply for photons. We then consider the process,

$$
q\left(p_{1}\right)+\bar{q}\left(p_{2}\right)+g\left(p_{3}\right)+g\left(p_{4}\right)+g\left(p_{5}\right) \rightarrow 0 .
$$

If we were to enumerate all $d$-dimensional tensor structures for this process, we would start noticing that, by stripping the amplitude of the gluon polarization vectors, we are left with a rank-3 Lorentz tensor, with one fermion line for the massless quarks. Each tensor will have the form,

$$
T \sim \epsilon_{3 \mu_{3}} \epsilon_{4 \mu_{4}} \epsilon_{5 \mu_{5}} \bar{u}\left(p_{2}\right) \Gamma^{\mu_{3} \mu_{4} \mu_{5}} u\left(p_{1}\right),
$$

where, in $d$ dimensions, the tensor $\Gamma^{\mu_{3} \mu_{4} \mu_{5}}$ can be built using combinations of Dirac $\gamma$ matrices, $g_{\mu \nu}$, and the external momenta $p_{i}^{\mu}, i=1, \ldots, 4$. Note that the maximum number of $\gamma$ matrices is, in general, given by the number of particles that can attach on the fermion line, which, in turn, depends on the number of loops. Of course, since we have only a finite number of contractions that we can do with the four independent external momenta, there is a finite number of 
such structures that we can build. Still, doing this exercise is very tedious and, as we have seen, not needed, since we only need to consider spinor chains that are independent in $d=4$ space-time dimensions.

Indeed, let us start considering the fermion lines $\bar{u}\left(p_{2}\right) \Gamma^{\mu_{3} \mu_{4} \mu_{5}} u\left(p_{1}\right)$. Their nontrivial part is given by the insertions of (arbitrary numbers of) $\gamma$ matrices,

$$
\bar{u}\left(p_{2}\right) \gamma^{\mu_{1}} \ldots \gamma^{\mu_{n}} u\left(p_{1}\right) .
$$

In order to see which structures are effectively allowed to show up in four dimensions, the crucial observation is that we can decompose any $\gamma$ matrix as

$$
\gamma^{\mu}=a_{1} p_{1}^{\mu}+\cdots+a_{4} p_{4}^{\mu},
$$

where the coefficients $a_{j}$ will, in general, depend on the external invariants and on all possible combinations of strings of $\not p_{i}$. Note that this is a nontrivial point. In fact, while the external states are assumed to be fourdimensional, the general prescription for 't HooftVeltman scheme implies that the $\gamma$ matrices in the spinor chain be $d$-dimensional. Nevertheless, one can show that when four-dimensional external states are considered, one only needs to consider spinor chains, which are independent when also all $\gamma$ matrices are in four dimensions (see Appendix B for a more detailed discussion). This implies that, for the purpose of finding a physical tensor basis, the only independent structures that we need to consider are of the form,

$$
T \sim \epsilon_{3 \mu_{3}} \epsilon_{4 \mu_{4}} \epsilon_{5 \mu_{5}} \bar{u}\left(p_{2}\right) \not p_{i_{1}} \ldots \not i_{n} u\left(p_{1}\right) p_{j_{3}}^{\mu_{3}} p_{j_{4}}^{\mu_{4}} p_{j_{4}}^{\mu_{4}} .
$$

Now, let us first focus on the fermion line. Clearly, the indices $i_{1}, \ldots, i_{n}$ can be only $i=3,4$; otherwise, we could anticommute the momenta to the left or to the right and use the Dirac equation to get rid of them. Also, since both $p_{1}$ and $p_{2}$ are massless, helicity conservation along the quark line implies that we can only have one occurrence of each, which limits the possible structures to

$$
T \sim \epsilon_{3 \mu_{3}} \epsilon_{4 \mu_{4}} \epsilon_{5 \mu_{5}} \bar{u}\left(p_{2}\right) \not \not_{3,4} u\left(p_{1}\right) p_{j_{3}}^{\mu_{3}} p_{j_{4}}^{\mu_{4}} p_{j_{5}}^{\mu_{5}} .
$$

A simple counting shows that these are $2 \times 4^{3}=128$ different structures. Now, we can use the physical constraints imposed by gauge invariance and transversality of the external gluons to show that many of these structure do not contribute. In particular, for each gluon, we can impose $\epsilon_{i} \cdot p_{i}=0$ and $\epsilon_{i} \cdot p_{j}=0$, leaving $2 \times 2^{3}=16$ structures. As expected, the number matches the number of helicity amplitudes for the process (two for the quark-line times 8 for the three external gluons). Once an explicit gauge choice is performed (or Ward identities are imposed), the corresponding 16 projectors can be obtained inverting a rather simple $16 \times 16$ matrix, which can be easily done with any computer algebra system.

It should be straightforward to see how to extend these considerations in the case of massive spin-1 external particles. For simplicity, we assume that we are working in a parity invariant theory as QCD or that, for other considerations, we expect the amplitude not to have any explicit dependence on parity-odd tensor structures. For each massive boson, we are allowed to impose only the transversality constrain $\epsilon_{i} \cdot p_{i}=0$, leaving one more possible choice for the momenta to contract the corresponding polarization vector. So, for example, if we are interested in the production of two massive vector bosons and a gluon in $q \bar{q}$ annihilation $q \bar{q} \rightarrow V V g$, we will be left with an upper bound of $2 \times 2 \times 3^{2}=36$ different tensor structures, corresponding to the 36 helicity amplitudes.

\section{B. Example 2: $q \bar{q} \rightarrow Q \bar{Q} g$ and $q \bar{q} \rightarrow Q \bar{Q} V$ scattering}

We conclude by showing what our method produces when we add an external massless or massive spin-1 particle to the $q \bar{q} Q \bar{Q}$ scattering considered in Sec. V. We remind the reader that this case was particularly interesting since one cannot find a finite number of tensor structures that span the full space if one insists in working in CDR. We showed instead that, by restricting the problem to four-dimensional external states, two tensors are enough to characterize the helicity amplitudes at any number of loops.

Let us start by studying what happens when we add an external massless gluon; i.e., we consider the process,

$$
q\left(p_{1}\right)+\bar{q}\left(p_{2}\right)+Q\left(p_{3}\right)+\bar{Q}\left(p_{4}\right)+g\left(p_{5}\right) \rightarrow 0 .
$$

As in the previous section, we start analyzing the fermion lines. We repeat the same arguments independently for the two fermion lines, and we find the only possible combinations in four dimensions,

$$
T \sim \epsilon_{5} \cdot p_{i} \bar{u}\left(p_{2}\right) \not p_{3,4} u\left(p_{1}\right) \bar{u}\left(p_{4}\right) \not p_{1,2} u\left(p_{3}\right) .
$$

Without imposing gauge invariance and transversality, these are $2 \times 2 \times 4=16$ different tensors, which become $2 \times 2 \times 2=8$ once we impose that the external gluon is onshell and physical; i.e., $\epsilon_{5} \cdot p_{5}=0$ plus one more condition to fix its gauge (or impose that the amplitude fulfils QCD Ward identities). Notice that, again, this matches the number of helicity amplitudes.

As a last case, we study what happens when the gluon is substituted by a massive external vector boson,

$$
q\left(p_{1}\right)+\bar{q}\left(p_{2}\right)+Q\left(p_{3}\right)+\bar{Q}\left(p_{4}\right)+V\left(p_{5}\right) \rightarrow 0 .
$$

Again, we assume that all parity-odd contributions coming from possible axial couplings of the massive vector bosons can be neglected. We stress here that, while this assumption 
is made only for exemplification purposes and similar arguments can be made also in the presence of axial couplings, it is often the case in massless QCD that axial contributions can be argued away by summing over degenerate isospin doublets. All considerations that lead us to the 16 tensors in Eq. (6.7) apply equally well here. The difference with the case of a massless gluon is that we can only impose one condition on the massive vector boson, namely transversality $\epsilon_{5} \cdot p_{5}=0$. We are, therefore, left with $2 \times 2 \times 3=12$ structures, which, of course, match the number of helicity amplitudes for the problem.

Finally, for both the massless and massive vector boson case, the explicit projectors can be derived by inverting very simple $8 \times 8$ or $12 \times 12$ matrices, respectively. The inversion can be obtained with any standard algebra system. As for the previous cases, we do not present an explicit choice here since, depending on the process analyzed, different choices of gauge can be more or less convenient.

\section{CONCLUSIONS}

In this paper, we proposed a new tensor decomposition for bosonic and femionic scattering amplitudes that allows us to combine naturally the form factor method usually defined in conventional dimensional regularization with the calculation of helicity amplitudes in 't Hooft-Veltman scheme. We have argued that, for an arbitrary number of external legs, one expects a natural correspondence between the number of independent helicity amplitudes in the process and the number of tensor structures that remain independent when four-dimensional external states are considered. We have called these tensors relevant tensors, and we have described a procedure that allows us to effectively decouple them from all remaining $d$-dimensional irrelevant tensors. While the latter would be required to obtain the value of the scattering amplitudes in CDR, we showed that they can be entirely neglected when computing the helicity amplitudes. A crucial property is that the relevant tensors must be chosen to span the entire vector space where the helicity amplitudes are defined. After describing the general idea, we have applied it to a large number of explicit examples for both bosonic and fermionic amplitudes with four and five external legs. The new projector operators are smooth in the limit $d \rightarrow 4$, and they are substantially simpler than the ones obtained using the standard approach in CDR. Importantly, the increase of the number of projectors with the number of legs is much slower than in CDR, being, in particular, bounded from above by the number of independent helicity amplitudes in the problem. Finally, the new projector operators can be applied on the scattering amplitudes in the very same way as the usual CDR projectors. We believe that the method proposed here constitutes an improvement compared to the commonly used approach and that it could prove useful, in the future, to compute complicated $2 \rightarrow 3$ scattering amplitudes, as well as lower-multiplicity amplitudes at higher loops.

\section{ACKNOWLEDGMENTS}

We thank F. Caola for many clarifying discussions at different stages of the project. The work of L. T. is supported by the Royal Society through Grant No. URF/ $\mathrm{R} 1 / 191125$.

\section{APPENDIX A: FINDING A PHYSICAL BASIS OF TENORS FOR $g g \rightarrow g g$}

In this paper, we have built a basis of physical tensors for several processes that are complemented by a set of irrelevant tensor, which can be ignored in the calculation of the helicity amplitudes. The physical tensors must, in particular, be a subset of the original tensors, or linear combinations thereof, that cover the full physical space spanned by the external polarizations. As explained in Sec. VI, for the scattering of $n \geq 5$ external particles, it is always straightforward to find such a basis by decomposing the tensor structures in terms of four independent external momenta. For $n=4$, instead, this is not always obvious. Therefore, we show here a more systematic approach based on the decomposition of momenta in the parallel and orthogonal space, which has already been successfully used in other contexts (see, e.g., Ref. [20]). In particular, we focus on the $g g \rightarrow g g$ example of Sec. III. Similar arguments can be applied to other four-point processes.

Because in four-point processes we have three linearly independent momenta, due to momentum conservation, we can split the physical space into a three-dimensional part spanned by the external momenta and an orthogonal part. Therefore, we can, in principle, cover the full physical space by adding a fourth four-dimensional momentum $v_{\perp}$, which is orthogonal to the external momenta $p_{j}$; e.g.,

$$
v_{\perp}^{\mu}=\epsilon^{\mu \nu \rho \sigma} p_{1 \nu} p_{2 \rho} p_{3 \sigma} .
$$

Hence, we may define physical tensors and projectors that only depend on scalar products of the form $\epsilon_{j} \cdot p_{k}$ and $\epsilon_{j} \cdot v_{\perp}$. One can also easily verify that a combination with an odd number of momenta $v_{\perp}$ is not allowed in a tensor, since it would vanish when contracted with the amplitude (this is also true in the presence of external fermions). A choice of eight independent physical tensors $\tilde{T}_{j}$ consistent with the gauge choices of Sec. III can be the following: 


$$
\begin{aligned}
& \tilde{T}_{1}=\epsilon_{1} \cdot p_{3} \epsilon_{2} \cdot p_{1} \epsilon_{3} \cdot p_{1} \epsilon_{4} \cdot p_{2}, \\
& \tilde{T}_{2}=\epsilon_{1} \cdot p_{3} \epsilon_{2} \cdot p_{1} \epsilon_{3} \cdot v_{\perp} \epsilon_{4} \cdot v_{\perp}, \\
& \tilde{T}_{3}=\epsilon_{1} \cdot p_{3} \epsilon_{3} \cdot p_{1} \epsilon_{2} \cdot v_{\perp} \epsilon_{4} \cdot v_{\perp}, \\
& \tilde{T}_{4}=\epsilon_{1} \cdot p_{3} \epsilon_{4} \cdot p_{2} \epsilon_{2} \cdot v_{\perp} \epsilon_{3} \cdot v_{\perp}, \\
& \tilde{T}_{5}=\epsilon_{2} \cdot p_{1} \epsilon_{3} \cdot p_{1} \epsilon_{1} \cdot v_{\perp} \epsilon_{4} \cdot v_{\perp}, \\
& \tilde{T}_{6}=\epsilon_{2} \cdot p_{1} \epsilon_{4} \cdot p_{2} \epsilon_{1} \cdot v_{\perp} \epsilon_{3} \cdot v_{\perp}, \\
& \tilde{T}_{7}=\epsilon_{3} \cdot p_{1} \epsilon_{4} \cdot p_{2} \epsilon_{1} \cdot v_{\perp} \epsilon_{2} \cdot v_{\perp}, \\
& \tilde{T}_{8}=\epsilon_{1} \cdot v_{\perp} \epsilon_{2} \cdot v_{\perp} \epsilon_{3} \cdot v_{\perp} \epsilon_{4} \cdot v_{\perp}
\end{aligned}
$$

In practice, however, the presence of $v_{\perp}$ would make the contractions with the amplitude unnecessarily involved. It is instead convenient to replace the terms involving orthogonal momentum $v_{\perp}$ with terms involving the metric tensor $g^{\mu \nu}$ so that we can replace the tensors $\tilde{T}_{j}$ with linear combinations of the original tensors $T_{j}$ defined in Eq. (3.4). We can do this by splitting the $d$ dimensional metric tensor $g^{\mu \nu}$ into a three-dimensional part $g_{[3]}^{\mu \nu}$, which is its projection into the subspace spanned by the external momenta, and an orthogonal $(d-3)$ dimensional part $g_{\perp}^{\mu \nu}$,

$$
g^{\mu \nu}=g_{[3]}^{\mu \nu}+g_{\perp}^{\mu \nu} .
$$

We can thus exploit the following identities:

$$
\begin{aligned}
v_{\perp}^{\mu} v_{\perp}^{\nu} & \sim g_{\perp}^{\mu \nu} \\
v_{\perp}^{\mu_{1}} v_{\perp}^{\mu_{2}} v_{\perp}^{\mu_{3}} v_{\perp}^{\mu_{4}} & \sim g_{\perp}^{\mu_{1} \mu_{2}} g_{\perp}^{\mu_{3} \mu_{4}}+g_{\perp}^{\mu_{1} \mu_{3}} g_{\perp}^{\mu_{2} \mu_{4}}+g_{\perp}^{\mu_{1} \mu_{4}} g_{\perp}^{\mu_{2} \mu_{3}},
\end{aligned}
$$

where the symbol $\sim$ indicates that the 1.h.s. and the r.h.s. become proportional when contracted with tensors depending on the $d$-dimensional metric $g^{\mu \nu}$ and the external momenta $p_{j}^{\mu}$ only. We note that the identities can be easily found (including the proportionality coefficient, which is irrelevant for the purposes of this paper) using standard tensor decomposition methods, although they must be valid also for symmetry reasons. The identities above allow us to replace tensors containing $v_{\perp}$, with suitable combinations of $g_{\perp}^{\mu \nu}$. Moreover, because $g^{\mu \nu}$ and $g_{\perp}^{\mu \nu}$ only differ by terms proportional to the external momenta, which are already accounted for in our basis of tensors, we are also allowed to replace $g_{\perp}^{\mu \nu}$ with $g^{\mu \nu}$. Putting everything together, this implies that, starting from the physical basis of tensors $\tilde{T}_{j}$ containing scalar products of the form $\epsilon_{j} \cdot v$, we can make the replacement,

$$
\epsilon_{j} \cdot v_{\perp} \epsilon_{k} \cdot v_{\perp} \rightarrow \epsilon_{j} \cdot \epsilon_{k},
$$

in any tensor containing only two scalar products involving $v_{\perp}$ that justifies replacing $\tilde{T}_{j} \rightarrow T_{j}$ for $j=1, \ldots, 7$.
For tensors with four scalar products involving $v_{\perp}$, we can similarly use the second Eq. (A4) to replace

$$
\tilde{T}_{8} \rightarrow T_{8}+T_{9}+T_{10},
$$

which then yields the same physical tensors defined in Eq. (3.7).

The same method can be applied to any process with $n \leq 4$ external legs. One considers a set of $5-n$ orthogonal vectors $v_{i, \perp}^{\mu}$ that span the $(5-n)$-dimensional subspace of the physical four dimensions that is orthogonal to the external legs. Thus, a basis of tensors is built assuming that they can be contracted only with external momenta $p_{i}^{\mu}$ or the vectors $v_{i, \perp}^{\mu}$. This is also true for the $\gamma$ matrices appearing inside spinor chains for the reasons illustrated in Appendix B. We note, again, that only combinations with an even number of orthogonal vectors need to be considered, since odd combinations would always give zero when contracted with a physical amplitude. Each combination of the form,

$$
v_{i_{1}, \perp}^{\mu_{1}} \cdots v_{i_{k}, \perp}^{\mu_{k}},
$$

can be rewritten in terms of the metric tensor $g_{\perp}^{\mu \nu}$, which is the restriction of $g_{\perp}^{\mu \nu}$ in the orthogonal space, and finally, we can replace $g_{\perp}^{\mu \nu} \rightarrow g^{\mu \nu}$ as explained above.

We also recall, from the discussion in Sec. VI, that these arguments are not needed with $n \geq 5$ external legs, since, in those cases, we can always use a subset of four independent external momenta to span the full physical space.

\section{APPENDIX B: SPINOR CHAINS WITH FOUR-DIMENSIONAL EXTERNAL STATES}

When dealing with external fermions, we need to build a basis of tensors involving spinor chains. In this Appendix, we show that, as stated, e.g., in Sec. V, when dealing with four-dimensional external states, we can limit ourselves to consider spinor chains that are independent when restricted to four dimensions. This is true despite the fact that the $\gamma$ matrices inside the spinor chain are $d$-dimensional objects, having a $d$-dimensional Lorentz index, with $d=4-2 \epsilon$.

Because, as we already mentioned, the algebra of the $\gamma$ matrices in $d$ dimensions is not closed [7], in principle, there is no limit to the allowed length of the spinor chains, and an infinite basis of tensors must therefore be considered. This is, in particular, relevant when two or more external fermion pairs are present because we can contract the Lorentz indices of gamma matrices belonging to two different spinor chains, which can, therefore, be arbitrarily long. In practice, at each loop order, one can always limit the length of the spinor chain to the maximum one that can appear in the diagrams of the process at that perturbative order, but this still yields a basis of tensors that is much larger than needed when dealing with four-dimensional 
external states. A different solution to this problem, which studies how extra-dimensional spinor indices decouple from the four-dimensional ones, has also been proposed in Ref. [21].

Let us consider a spinor chain of length $n$ of the form,

$$
\bar{u}\left(p_{1}\right) \gamma^{\mu_{1}} \gamma^{\mu_{2}} \cdots \gamma^{\mu_{n}} u\left(p_{2}\right),
$$

although replacing a spinor $u$ with an antispinor $v$ doesn't change our conclusions. Here, we consider the spinors in Eq. (B1) to be four-dimensional, but the $\gamma$ matrices to be instead $d$-dimensional objects, with a $d$-dimensional Lorentz index, satisfying the usual anticommutation relations,

$$
\left\{\gamma^{\mu}, \gamma^{\nu}\right\}=2 g^{\mu \nu} \mathbb{1},
$$

where $g^{\mu \nu}$ is the $d$-dimensional metric tensor. In particular, the Lorentz indices in Eq. (B1) can be assumed to be contracted with either external momenta and polarization vectors or with Lorentz indices belonging to other spinor chains. We can thus split, for each $\gamma$ matrix, contributions from four-dimensional and $(d-4)$-dimensional indices with

$$
\gamma^{\mu}=\gamma_{[4]}^{\mu}+\gamma_{[-2 \epsilon]}^{\mu},
$$

where $\gamma_{[4]}^{\mu}\left(\gamma_{[-2 \epsilon]}^{\mu}\right)$ is an object that is equal to $\gamma^{\mu}$ if $\mu$ is a four-

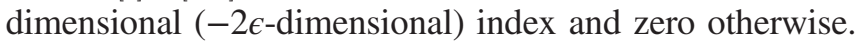
We obviously have

$$
\left\{\gamma_{[4]}^{\mu}, \gamma_{[-2 \epsilon]}^{\nu}\right\}=0
$$

which allows us to anticommute all the $\gamma_{[-2 \epsilon]}^{\mu}$ matrices to one side of the spinor chain. This way, up to a relabeling of the indices, the chain in Eq. (B1) becomes a linear combination of contributions of the form,

$$
\bar{u}\left(p_{1}\right) \gamma_{[-2 \epsilon]}^{\mu_{1}} \cdots \gamma_{[-2 \epsilon]}^{\mu_{n_{1}}} \gamma_{[4]}^{\nu_{1}} \cdots \gamma_{[4]}^{\nu_{n_{2}}} u\left(p_{2}\right) .
$$

with $n_{1}+n_{2}=n$. We can now perform a tensor decomposition of Eq. (B5) with respect to its free (Lorentz) indices. In particular, we can do so in terms of a basis of four-dimensional vectors $\left\{e_{j}^{\mu}\right\}_{j=1}^{4}$ and the $(-2 \epsilon)$-metric tensor $g_{[-2 \epsilon]}^{\mu \nu}$. Because the four-dimensional and $(-2 \epsilon)$ dimensional parts are now completely decoupled from each other, we can also limit ourselves to perform a tensor decomposition of the $\gamma_{[-2 \epsilon]}^{\mu}$ parts alone, in terms of $g_{[-2 \epsilon]}^{\mu \nu}$ only. By doing that, we obtain

$$
\begin{array}{r}
\bar{u}\left(p_{1}\right) \gamma_{[-2 \epsilon]}^{\mu_{1}} \cdots \gamma_{[-2 \epsilon]}^{\mu_{n_{1}}} \gamma_{[4]}^{\nu_{1}} \cdots \gamma_{[4]}^{\nu_{n_{2}}} u\left(p_{2}\right) \\
=c^{\mu_{1} \cdots \mu_{n_{1}}} \bar{u}\left(p_{1}\right) \gamma_{[4]}^{\nu_{1}} \cdots \gamma_{[4]}^{\nu_{n_{2}}} u\left(p_{2}\right),
\end{array}
$$

where $c^{\mu_{1} \cdots \mu_{n_{1}}}$ only depends on $g_{[-2 \epsilon]}^{\mu \nu}$ and $\epsilon$. The coefficients $c^{\mu_{1} \cdots \mu_{n_{1}}}$ obviously vanish when contracted with fourdimensional vectors, but they can give a nonvanishing $d$-dependent contribution when contracted with metric tensors, coming, e.g., from similar coefficients in other spinor chains.

We have thus shown that any $d$-dimensional spinor chain of length $n$, when the external states are four-dimensional, can be rewritten in terms of purely four-dimensional spinor chains (where also the $\gamma$ matrices are in four dimensions) with length $n_{1} \leq n$. It is therefore easy to show (e.g., by induction in the length $n$ ) that spinor chains in $d$ dimensions, when contracted with four-dimensional external spinors, are linearly independent if and only if they are independent in four dimensions. When building a physical basis of tensors, we are therefore allowed to consider only spinor chains that are linearly independent when restricted to $d=4$.
[1] C. Bollini and J. Giambiagi, Dimensional renormalization: The number of dimensions as a regularizing parameter, Nuovo Cimento B 12, 20 (1972).

[2] G. Cicuta and E. Montaldi, Analytic renormalization via continuous space dimension, Lett. Nuovo Cimento 4, 329 (1972).

[3] G. 't Hooft and M. J. G. Veltman, Regularization and renormalization of gauge fields, Nucl. Phys. B44, 189 (1972).

[4] C. Gnendiger et al., To $d$, or not to $d$ : Recent developments and comparisons of regularization schemes, Eur. Phys. J. C 77, 471 (2017).
[5] L. Garland, T. Gehrmann, E. Glover, A. Koukoutsakis, and E. Remiddi, Two loop QCD helicity amplitudes for $e^{+} e^{-} \rightarrow$ three jets, Nucl. Phys. B642, 227 (2002).

[6] E. Glover and M. Tejeda-Yeomans, Two loop QCD helicity amplitudes for massless quark massless gauge boson scattering, J. High Energy Phys. 06 (2003) 033.

[7] E. Glover, Two loop QCD helicity amplitudes for massless quark quark scattering, J. High Energy Phys. 04 (2004) 021).

[8] T. Gehrmann, A. von Manteuffel, and L. Tancredi, The twoloop helicity amplitudes for $q \bar{q}^{\prime} \rightarrow V_{1} V_{2} \rightarrow 4$ leptons, J. High Energy Phys. 09 (2015) 128. 
[9] L. Chen, A prescription for projectors to compute helicity amplitudes in D dimensions, arXiv:1904.00705.

[10] T. Ahmed, A. Ajjath, L. Chen, P. K. Dhani, P. Mukherjee, and V. Ravindran, Polarised amplitudes and soft-virtual cross sections for $b \bar{b} \rightarrow Z H$ at NNLO in QCD, J. High Energy Phys. 01 (2020) 030.

[11] T. Peraro and L. Tancredi, Physical projectors for multi-leg helicity amplitudes, J. High Energy Phys. 07 (2019) 114.

[12] T. Binoth, E. Glover, P. Marquard, and J. van der Bij, Two loop corrections to light by light scattering in supersymmetric QED, J. High Energy Phys. 05 (2002) 060.

[13] A. Hodges, Eliminating spurious poles from gauge-theoretic amplitudes, J. High Energy Phys. 05 (2013) 135.

[14] S. Badger, Automating QCD amplitudes with on-shell methods, J. Phys. Conf. Ser. 762, 012057 (2016).

[15] F. Caola, J. M. Henn, K. Melnikov, A. V. Smirnov, and V. A. Smirnov, Two-loop helicity amplitudes for the production of two off-shell electroweak bosons in gluon fusion, J. High Energy Phys. 06 (2015) 129.
[16] A. von Manteuffel and L. Tancredi, The two-loop helicity amplitudes for $g g \rightarrow V_{1} V_{2} \rightarrow$ 4leptons, J. High Energy Phys. 06 (2015) 197.

[17] J. Davies, G. Mishima, M. Steinhauser, and D. Wellmann, $g g \rightarrow Z Z$ : Analytic two-loop results for the low- and highenergy regions, J. High Energy Phys. 04 (2020) 024.

[18] F. Caola, A. von Manteuffel, and L. Tancredi, Di-Photon Amplitudes in Three-Loop Quantum Chromodynamics, arXiv:2011.13946 [Phys. Rev. Lett. (to be published)].

[19] T. Ahmed, J. Henn, and B. Mistlberger, Four-particle scattering amplitudes in QCD at NNLO to higher orders in the dimensional regulator, J. High Energy Phys. 12 (2019) 177.

[20] P. Mastrolia, T. Peraro, and A. Primo, Adaptive integrand decomposition in parallel and orthogonal space, J. High Energy Phys. 08 (2016) 164.

[21] S. Abreu, F. F. Cordero, H. Ita, B. Page, and V. Sotnikov, Planar two-loop five-parton amplitudes from numerical unitarity, J. High Energy Phys. 11 (2018) 116. 